\title{
Do Patients With Irritable Bowel Syndrome Exhale More Hydrogen Than Healthy Subjects in Fasting State?: Author's Reply
}

TO THE EDITOR: We appreciate the interest of Dr. Choi on our paper and for their critical appraisal of our results. As seen in the figure provided in our previous study, healthy subject (HS) had lowest value of hydrogen excretion in fasting state compared to patients with chronic non-specific diarrhea (CNSD) and irritable bowel syndrome (IBS). ${ }^{1}$ Though the difference between fasting breath hydrogen excretion in HS and patients with IBS was not significant in that study, ${ }^{1}$ it is worthwhile to realize that most of those who had diarrhea in absence of malabsorption might be patients with diarrheal IBS. Therefore, it is important to club patients with CNSD and IBS in the perspective of our second study. ${ }^{1}$ Such an analysis showed that fasting breath hydrogen lev$\mathrm{el}$ in $50 \mathrm{HS}$ tended to be lower than that in 204 patients with IBS and CNSD $(4.9 \pm 5.4$ vs $6.5 \pm 6.3$ ppm, p $=0.08){ }^{1}$

Dr. Choi suggested that the results of the present study comparing fasting breath hydrogen between constipation and diarrhea predominant IBS might have changed if we classified the IBS subtypes by Rome III criteria. ${ }^{2}$ We beg to differ on this issue. There are several reports, which showed that sub-typing IBS using Rome III criteria may not be appropriate in Asia in general and in India in particular. ${ }^{3-5}$ Though $95 \%$ patients in our study reported looser stools at onset of pain, this symptom is not the only determinant to subtype IBS. ${ }^{2}$ We reiterate that another study done previously, had limitations as they included non comparable patients with IBS and HS. ${ }^{6}$ However, we fully agree with Dr. Choi that further studies with a larger sample size are needed on this issue. In fact, we believe that many of the gastrointestinal diseases which were once thought to be psychogenic, later turned out to be related to structural or functional defect in the gastrointestinal system. ${ }^{5}$ Similarly, bloating in patients with IBS might be related to distension of the gut with gas such as hydrogen. 3,5 Since $96 \%$ of patients in our study had bloating and only $4 \%$ did not report it, we could not study the relationship between bloating and fasting breath hydrogen. ${ }^{2}$ Future studies should be planned to evaluate the relationship between patient-perceived bloating, and that measured using playthismography and fasting breath hydrogen on an adequate number of patients.

We agree with Dr. Choi that repeated toilet visit by the patients with constipation predominant IBS may result in spuriously increased stool frequency. Hence, we reanalyzed our data using Bristol Stool Form as suggested; we found that there was correlation between fasting breath hydrogen excretion and number of stools per week among patients with IBS who were passing Bristol Stool Form 5 and $6(r=0.35, p=0.02)$. However, there was no correlation between fasting breath hydrogen excretion and number of stool per week among the patients passing Bristol Stool Form 1 to 4.

We appreciate the interest of Dr. Choi on this subject in general and on our paper in particular. We believe that inspite of the limitation of our study, it attempted to explore a new issue of whether excess hydrogen production in the gut even in fasting state by the patients with IBS may explain their symptom at least partly. We hope this may give a new insight on this disease which is left in the realm of psychogenic nature rather than an abnormal primary gastrointestinal abnormality.

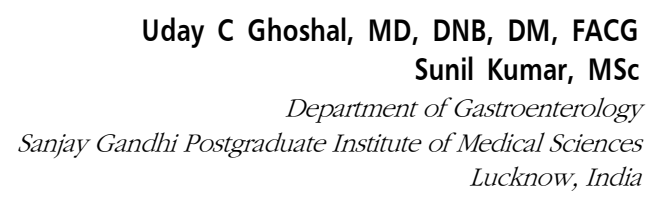

1. Ghoshal UC, Kumar S, Mehrotra M, Lakshmi C, Misra A. Frequency of small intestinal bacterial overgrowth in patients with irritable bowel syndrome and chronic non-specific diarrhea. J Neurogastroenterol Motil 2010;16:40-46.

2. Kumar S, Misra A, Ghoshal UC. Patients with irritable bowel syn- 
drome exhale more hydrogen than healthy subjects in fasting state. J Neurogastroenterol Motil 2010;16:299-305.

3. Gwee KA, Bak YT, Ghoshal UC, et al. Asian consensus on irritable bowel syndrome. J Gastroenterol Hepatol 2010;25:1189-1205.

4. Ghoshal UC, Abraham P, Bhatt C, et al. Epidemiological and clinical profile of irritable bowel syndrome in India: report of the Indian Society of Gastroenterology Task Force. Indian J Gastroenterol 2008; 27:22-28.

5. Ghoshal UC, Park H, Gwee KA. Bugs and irritable bowel syn- drome: the good, the bad and the ugly. J Gastroenterol Hepatol 2010;25:244-251.

6. Bratten JR, Spanier J, Jones MP. Lactulose breath testing does not discriminate patients with irritable bowel syndrome from healthy controls. Am J Gastroenterol 2008;103:958-963.

\section{Conflicts of interest: None.}

